\title{
A Generalized Minimum Cost Flow Model for Multiple Emergency Flow Routing
}

\author{
Jianxun Cui, Shi An, and Meng Zhao \\ School of Transportation Science and Engineering, Harbin Institute of Technology, Harbin 150090, China \\ Correspondence should be addressed to Shi An; anshi@hit.edu.cn
}

Received 29 March 2014; Accepted 27 May 2014; Published 22 June 2014

Academic Editor: Michael Lütjen

Copyright ( 2014 Jianxun Cui et al. This is an open access article distributed under the Creative Commons Attribution License, which permits unrestricted use, distribution, and reproduction in any medium, provided the original work is properly cited.

\begin{abstract}
During real-life disasters, that is, earthquakes, floods, terrorist attacks, and other unexpected events, emergency evacuation and rescue are two primary operations that can save the lives and property of the affected population. It is unavoidable that evacuation flow and rescue flow will conflict with each other on the same spatial road network and within the same time window. Therefore, we propose a novel generalized minimum cost flow model to optimize the distribution pattern of these two types of flow on the same network by introducing the conflict cost. The travel time on each link is assumed to be subject to a bureau of public road (BPR) function rather than a fixed cost. Additionally, we integrate contraflow operations into this model to redesign the network shared by those two types of flow. A nonconvex mixed-integer nonlinear programming model with bilinear, fractional, and power components is constructed, and GAMS/BARON is used to solve this programming model. A case study is conducted in the downtown area of Harbin city in China to verify the efficiency of proposed model, and several helpful findings and managerial insights are also presented.
\end{abstract}

\section{Introduction}

Unfortunately, real-life situations such as floods, hurricanes, chemical accidents, nuclear accidents, terrorist attacks, and other events may occur and threaten the lives and the health of human beings [1]. Evacuation and emergency rescue are two major activities in disaster response. Evacuation involves relocating the threatened populations to safer areas (i.e., shelters) as soon as possible, whereas emergency rescue operations aim to dispatch various special vehicles (i.e., police cars, fire trucks, ambulances, etc.) to save lives, mitigate emergency situations, or conduct other special operations. These two types of flow will inevitably conflict with each other during the emergency response phase because they share the same spatial road network during the same time window. Usually, emergency vehicles have a higher priority to obtain the right-of-way when they share the same link or intersection with evacuation vehicles. Evacuation vehicles and other public vehicles must be stopped to reduce response times and enhance traffic safety when a rescue vehicle approaches. As many researchers have claimed, when evacuation is implemented, the evacuation traffic demand will surge into the capacitated road network over a short period of time and consequently may cause significant transportation problems. Traffic delays during evacuation may range from inconvenient to catastrophic. Clearly, traffic delays will further be aggravated if evacuation flow and rescue flow conflict with each other in the same spatial and temporal space, especially when evacuation vehicles on the road may be often required to yield the right-of-way to rescue vehicles that use their warning devices. Therefore, emergency managers face a serious and difficult problem, namely, how to establish collaboration between evacuation flow and rescue flow in a shared and capacitated road network to mitigate negative influence due to conflicts. An obvious solution is to completely separate these two types of flow using selected management policies, such as setting up special emergency lanes for rescue vehicles to pass. The major drawback of this effort may be that the network capacity will not be fully explored because mixing of two flows is absolutely excluded. Thus, emergency managers must not only reduce conflict between two flows but also waste as little network capacity as possible.

The other issue commonly faced by emergency managers is how to implement contraflow design (also referred to 
as lane reversal operations) during emergency response to fully explore the capacity of the current network. Contraflow design commonly refers to the shift of the normal driving directions of a subset or all danger-bound lanes for use by safety-bound evacuation traffic. Such control is based on the observation that danger-bound traffic is usually light, whereas evacuation traffic always oversaturates the safetybound capacity. In nature, contraflow design constitutes a network redesign problem. If evacuation flow and rescue flow exist together in the same network, we can reshape the network to better serve these two flows.

Based on the previous discussion, this paper aims to present a generalized minimum cost flow model for collaboration between evacuation flow and rescue flow that also accounts for optimal contraflow design in a complex road network.

The paper is organized as follows. We first review related prior work, and the subsequent section presents the model formulation. The Baron solver is used to solve the nonconvex mixed-integer nonlinear programming model, and a local network in downtown Harbin City, Heilongjiang, China, is adopted to implement the case study. The paper concludes with a discussion of the results and areas for further research.

\section{Literature Review}

Over the past several decades, a wide range of network flow models has been introduced to describe different versions of routing and evacuation problems. The maximum dynamic flow problem introduced by Ford and Fulkerson [2] can be easily interpreted in the evacuation context as the evacuation of as many people as possible from a danger zone (source node) into a safe zone (sink node). The earliest arrival flow problem presented by Gale [3] is an extension of the maximum dynamic flow problem. The earliest arrival flow translated into the evacuation context means that the maximal amount of evacuees enters the safe area in each time period. To represent the evolution of a building evacuation process over time, Chalmet et al. [4] constructed a dynamic network flow model by expanding the network into a timespace network. The objective is to minimize the time until the last evacuee exits, which is known as the quickest flow problem. Following the same line of inquiry, Hamacher and Tufekci [5] extended the quickest flow problem to take into account different priority levels for different components of the evacuation network. Choi et al. [6] formulated three dynamic network flow problems for building evacuation (i.e., maximum flow, minimum cost, and the quickest flow problems) and introduced additional constraints to define link capacity as a function of the incoming flow rate. By formulating evacuation routing as a minimum cost flow problem, Dunn and Newton [7] proposed two algorithms for finding the set of path flows that minimize the total travel distance through a capacity-constrained network. Cova and Johnson [8] proposed the concept of lane-based routing to reduce intersection delays by temporarily transforming intersections into uninterrupted flow facilities using proper turning restrictions. As an extension of the minimal cost flow problem, the model minimizes the total travel distance while preventing flow conflicts and restricting merge points at intersections. Miller-Hooks and Patterson [9] proposed the time-dependent quickest flow problem in time-varying capacitated evacuation networks in which link travel times and capacities vary with time. Network flow is modeled with flow conservation constraints at each node as well as link capacity constraints. As an extension of the timedependent quickest flow problem, Opasanon [10] addressed the stochastic nature of the evacuation network for a large building and formulated two network flow problems to generate the optimal path flows. The minimal cost problem seeks to minimize the total travel time if both link capacities and travel time are random variables with time-varying probability mass functions. In contrast, the safest escape problem aims to maximize the minimum path probability of successful arrivals at destinations on a network with a deterministic travel time and stochastic time-varying link capacities. Recently, Bretschneider and Kimms [1] presented a basic mathematical model for evacuation problems based on time-expanded network flow model that minimizes the evacuation time and prohibits conflicts within intersections.

Besides using network flow model to produce origindestination routes and schedules of evacuees on each route, traffic assignment-simulation approaches are also employed to model evacuation problems. These approaches often use static/dynamic, deterministic/stochastic traffic assignment models to get evacuation flow pattern on road network (e.g., $[11,12]$ ) and then use traffic simulation tools, such as DYNASMART [13] and DynaMIT [14], to conduct stochastic simulation of traffic movements based on origin-destination traffic demands and use queuing methods to account for road capacity constraints. However, it may take a long time to complete the simulation process for a large transportation network.

Lane reversal, which is also known as a contraflow lane, is another common topic during evacuation modeling. The effectiveness, feasibility, and safety issues of implementing lane reversal have been extensively discussed in, for example, MacDorman [15], Glickman [16], Hemphill and Surti [17], and Caudill and Kuo [18]. In evacuation cases, it has been suggested that the traffic direction of the inbound lanes of eligible roadway segments may be reversed in the case of an overwhelming flow of outbound traffic to increase the outbound capacity. Since the late 1990s, lane reversal has been widely used for hurricane evacuations in the states of the U.S. located on the Atlantic and Gulf Coasts [19]. The results from both evacuation practices $[19,20]$ and numerical studies [21-24] show that lane reversal has great potential to enhance evacuation performance and reduce traffic delays. It is worth noting that Xie and Turnquist [25] presented a lanebased evacuation bilevel programming model that integrates lane reversal and crossing elimination strategies in which network redesign and evacuation flow modeling are perfectly integrated. However, this research only aims to minimize the total evacuation time without considering that there may be multiple emergency flows that conflict with each other.

In summary, prior studies primarily formulate the evacuation networks in terms of facilities with limited capacity 
in which traffic can travel through links with known travel times if they do not exceed the link capacity. These problems typically involve two types of network flow constraints, namely, flow conservation constraints at every node and capacity constraints for each link. However, certain traffic phenomena, for example, congestion-caused delays, are not captured in such models. Additionally, evacuation flow does not independently move during the emergency response phase and usually coincides with emergency rescue flow in the same spatial and temporal framework. Ensuring that all flows collaborate together could be a more practical step for the emergency management field. Few researches have been conducted to investigate multiple flow conflict during evacuation flow modeling and integrating contraflow lane design together, except the research presented by Xie and Turnquist [26], which integrated reversing lanes, eliminating intersection crossings, and reserving lanes for use by emergency vehicles together into one model. Although reserving a specific lane for emergency vehicles' exclusive use would improve the rescue efficiency, it may cause some extent of waste of road capacity, since the reserving lanes cannot be used for evacuation even though rescue flow is relatively low. In our research, instead of separating evacuation flow and rescue flow absolutely, we define conflict cost to account for the result of two flows mixing, which can be regarded as a general extension of Xie and Turnquist research [26].

\section{Model Formulation}

In this section, we model multiple emergency flow routing (MEFR). It is assumed that, in a specified emergency scenario, evacuation and rescue operations are both necessary, and contraflow design also can be implemented to explore further capacity of original road network in an emergency area. The emergency manager seeks to maximize the efficiency of the emergency response. The mathematical notation used in our models is introduced in Notation summary.

Before developing the model formulation, we first present selected illustrations of road network representation. In a specified emergency response network, several evacuation origins and destinations may exist as well as several rescue origins and destinations. The original network can be augmented with "virtual links" leading from each realworld origin/destination point to one common virtual origin/destination point [27]. All these virtual links are assumed to have infinite capacity and zero cost (i.e., zero travel time) so as not to influence the flow routing.

The MEFR problem described in this section is based on the minimum cost flow problem [28]. The min-cost flow problem occupies a central position among the network optimization models because it encompasses a broad class of applications [29]. The objective is to minimize the cost of transporting all supply (source nodes) to meet all demand (destination nodes) in a capacitated network. The MEFR model is an extension of min-cost flow problem. The objective of MEFR is to minimize the four components of the cost, that is, evacuation-flow time cost, rescue-flow time cost, conflict cost, and lane reversal cost.
During emergency operations, evacuation flow and rescue flow may conflict with each other, and we define this conflict as follows.

Conflicting Flows. If evacuation flow and emergency flow coexist on a same road link, they are treated as conflicting flows.

The inspiration for this definition originates from events that often occur during emergency response. If a rescue flow mixes with the evacuation flow, significant traffic delay may result. For example, if an emergency vehicle is required to rapidly pass through a link, evacuation flow on that link must be stopped or slowed to avoid conflict. It is usually true that rescue flow has a higher level of priority than evacuation flow, whereas evacuation flow possesses a relatively higher demand. It can be observed that this type of conflict (two types of flow coexisting on the same links) can cause large traffic delays or even serious disorder. The resulting cost due to emergency flow conflict should be proportional to the size of the coexisting evacuation and rescue flow on a specified link $(i, j) \in A$, which is presented as follows:

$$
H=\theta \sum_{(i, j) \in A} x_{i j} y_{i j},
$$

where $H$ is the total conflict cost resulting from two types of flow. For special cases, when $x_{i j}=0$ (that is no evacuation flow on link $(i, j)$ ) or $y_{i j}=0$ (that is no rescue flow on link $(i, j))$, the conflict cost on link $(i, j)$ will be zero.

There are also two components of time cost during emergency response: the time cost of evacuation flow and the time cost of rescue flow, which are determined by formulas (2):

$$
\begin{aligned}
& E=\lambda_{1} \sum_{(i, j) \in A} t_{i j} x_{i j}, \\
& P=\lambda_{2} \sum_{(i, j) \in A} t_{i j} y_{i j},
\end{aligned}
$$

where $E$ is the total evacuation time cost and $P$ is the total rescue time cost. In a classical min-cost flow problem, the travel times of the links are assumed to be constant, but, for road transportation problems, this assumption should be relaxed because the travel speed of the link will decrease together with the increase in link traffic flow. To account for this traffic phenomenon, we introduce the BPR function [30] to describe the link travel time.

The final component of cost is the lane reversal cost. It should be noted that during contraflow lane design, two links are required for use in operations, one for each direction. These two links are referred to as a "pair" of links, and operators will not reverse them concurrently [31]. Normally, each road will contain two links serving two different directions, and, naturally, they will be considered as paired links. If a given road is one way (i.e., only one link), we can imagine that there is also a virtual link serving the opposite direction with zero capacity, and these consequently will become paired links. Contraflow lane operations can also incur costs because human resources and certain essential devices are needed to 
manage the contraflow lane to avoid confusing drivers. This cost should be proportional to the number of reversal lanes and is described as follows:

$$
M=\frac{\delta \sum_{(i, j) \in A}\left|\mathrm{NL}_{i j}-\gamma_{i j}\right|}{2},
$$

where $M$ is the total line reversal cost and $\delta\left|\mathrm{NL}_{i j}-\gamma_{i j}\right|$ is the lane reversal cost shared by link $(i, j)$ and its pair link $(j, i)$.

Based on the discussion of the four components of emergency cost, a programming model is presented to describe the MEFR problem by extending the minimal cost flow as follows (MEFR):

$$
\begin{aligned}
\min _{x, y, \gamma} z= & \lambda_{1} \sum_{(i, j) \in A} t_{i j} x_{i j}+\lambda_{2} \sum_{(i, j) \in A} t_{i j} y_{i j}+\theta \sum_{(i, j) \in A} x_{i j} y_{i j} \\
& +\frac{\delta \sum_{(i, j) \in A}\left|\mathrm{NL}_{i j}-\gamma_{i j}\right|}{2}
\end{aligned}
$$

s.t.

$$
\begin{aligned}
& \sum_{j \in \xi(i)} x_{i j}-\sum_{k \in \zeta(i)} x_{k i}=m_{i}, \quad \forall i \in V \\
& \sum_{j \in \xi(i)} y_{i j}-\sum_{k \in \zeta(i)} y_{k i}=n_{i}, \quad \forall i \in V
\end{aligned}
$$

$m_{i}= \begin{cases}+d_{1}, & \text { if } i \text { is the virtual evacuation origin node } \\ 0, & \text { if } i \text { is an intermediate transfer node } \\ -d_{1}, & \text { if } i \text { is the virtual evacuation sink node }\end{cases}$

$n_{i}= \begin{cases}+d_{2}, & \text { if } i \text { is the virtual rescue origin node } \\ 0, & \text { if } i \text { is an intermediate transfer node } \\ -d_{2}, & \text { if } i \text { is the virtual rescue sink node }\end{cases}$

$$
\begin{gathered}
\gamma_{i j}+\gamma_{j i}=\mathrm{NL}_{i j}+\mathrm{NL}_{j i}, \quad \forall(i, j),(j, i) \in A \\
x_{i j}+y_{i j} \leq \bar{\mu}_{i j}, \quad \forall(i, j) \in A \\
t_{i j}=t_{i j}^{0} \times\left[1+\alpha\left(\frac{x_{i j}+y_{i j}}{\bar{\mu}_{i j}}\right)^{\beta}\right] \\
\bar{\mu}_{i j}=\frac{\left(\gamma_{i j} \times \mu_{i j}\right)}{\mathrm{NL}_{i j}} \\
x_{i j}=S_{j}^{e, o}, \quad i=O_{e}^{*}, \quad \forall j \in O_{e} \\
x_{i j}=R_{j}^{e, d}, \quad i=D_{e}^{*}, \quad \forall j \in D_{e} \\
y_{i j}=S_{j}^{r, o}, \quad i=O_{r}^{*}, \quad \forall j \in O_{r} \\
y_{i j}=R_{j}^{r, d}, \quad i=D_{r}^{*}, \quad \forall j \in D_{r} \\
x_{i j} \geq 0, \quad y_{i j} \geq 0 ; \quad \forall(i, j) \in A \\
\gamma_{i j} \in \mathrm{Integer}^{2} \quad \forall(i, j) \in A .
\end{gathered}
$$

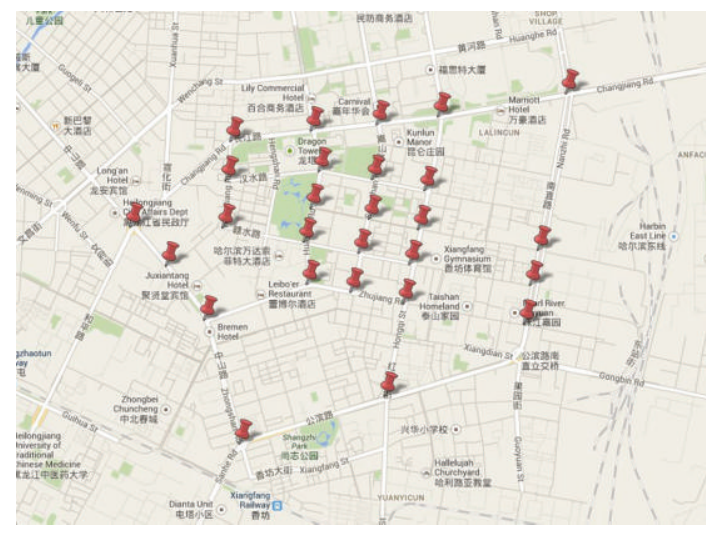

FIGURE 1: Road network for the case study.

In MEFR, $x_{i j}, y_{i j}$, and $\gamma_{i j}$ for each $(i, j) \in A$ are decision variables. The objective of (1) is to minimize the total cost composed of the four components of cost in which $t_{i j}$ is defined in Constraint (11). Constraint (5) is the evacuation flow conservation at each node, and Constraint (6) is the rescue flow conservation at each node in which $m_{i}$ (evacuation flow at node $i$ ) and $n_{i}$ (rescue flow at node $i$ ) are defined by Constraints (7) and (8). Constraint (9) guarantees that the summation of the lane numbers of two arbitrary "paired" links after contraflow design is equal to the summation of lane numbers before contraflow design. Constraint (10) illustrates that the summation of two flows on each link is always not greater than the link capacity after contraflow design in which $\bar{\mu}_{i j}$ is defined by Constraint (12). Constraints (13)-(16) define the flow of virtual links to guarantee that each evacuation/rescue origin can send a specified amount of flow, and each evacuation/rescue destination can receive a specified amount of flow. Constraint (17) states that the evacuation flow and rescue flow on each link are nonnegative. Constraint (18) means that the number of lanes on each link after the contraflow design must be an integer.

The MEFR is relatively difficult to solve because it is a nonconvex mixed-integer nonlinear programming (nonconvex MINLP) model with bilinear, fractional, and power components. The MINLP problems are difficult to solve because they combine all the difficulties of both of their subclasses: the combinatorial nature of mixed-integer programs (MIP) and the difficulty of solving nonconvex (and even convex) nonlinear programs (NLP). Because subclasses MIP and NLP are among the class of theoretically difficult problems (NPcomplete), it is not surprising that solving the MINLP can be a challenging and daring venture [32]. In this paper, the branch-and-reduce optimization navigator (BARON), that is, a GAMS solver for the global solution of nonlinear (NLP) and mixed-integer nonlinear programs (MINLP), is used to solve the MEFR model.

\section{Case Study}

This section presents a case study of the MEFR model. The study area consists of the downtown area of Nangang 


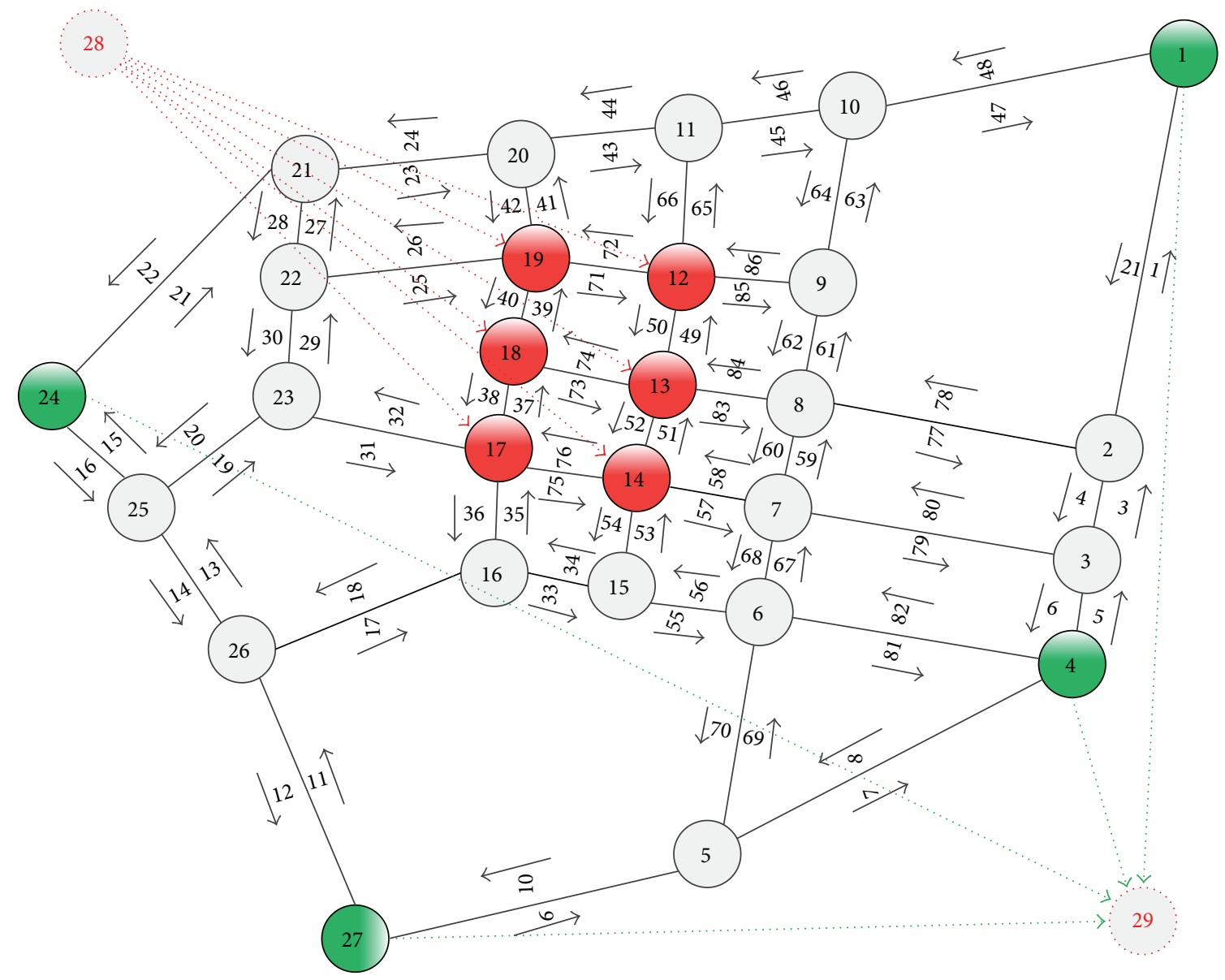

FIGURE 2: Network representation with virtual nodes and links.

District, Harbin City, China, which contains 27 intersections and 86 links (see Figure 1). This area includes a large-scale comprehensive international exhibition and sports center, the Heilongjiang Province TV Station, Wanda Plaza (the city's comprehensive business center), large movie centers, shopping malls, hotels, hospitals, and many residential areas. Additionally, the density of roads is quite large in this area, including many main roads. The area is capable of generating many thousands of vehicle trips during a daytime evacuation and is therefore suitable for testing our model.

As stated in Section 3, we must transform the real-world road network into an arc-node network that integrates the virtual evacuation/rescue origin and the evacuation/rescue destination (see Figure 2). In Figure 2, the nodes shown in red (i.e., 12, 13, 14, 17, 18, and 19) are the hypothetical real-world evacuation/rescue origins, whereas the green nodes are the hypothetical real-world evacuation/rescue destinations (i.e., $1,4,24$, and 27). In addition, the virtual origin node (i.e., 28) and the virtual destination node (i.e., 29) are introduced to generate and receive evacuation/rescue flows. All roads are bidirectional, and two links are paired for potential use in contraflow operations. The link IDs are depicted in Figure 2, and the characteristics of each link are presented in Table 1.

The evacuation flow demand and rescue flow demand in this hypothetical emergency scenario are presented in
Table 2 in which a positive number indicates origin flow and a negative number denotes sink flow.

We conducted four groups of experiments to test our model. In the first two groups, contraflow lane design and flow conflict are not considered together, that is, only contraflow lane or flow conflict is investigated independently in our model, whereas these flows are combined together in the other two groups. We aim to explore how the variations of the unit cost of lane reversal operations and flow conflict affect the four components of cost and total cost.

Group 1. In this group of tests, we do not consider flow conflict, which means that we do not emphasize the separation of evacuation flow and rescue flow. We aim to investigate how the unit cost of lane reversal operations affects the evacuation cost, rescue cost, lane reversal cost, and total cost. We define the values of the parameters as follows. The unit evacuation $\operatorname{cost} \lambda_{1}=1$, the unit rescue cost $\lambda_{2}=3$, and the unit lane reversal cost $\delta$ increases 200 times from 0.1 in step sizes of 0.4 . The results are shown in Figure 3.

We observe that, after the unit lane reversal cost reaches slightly more than 40 , the lane reversal cost stabilizes at zero, and the evacuation cost, rescue cost, and total cost also stabilize at certain values. This result indicates that when the lane reversal cost becomes too large (i.e., larger than 40), we 
TABLE 1: Characteristics of links.

\begin{tabular}{|c|c|c|c|c|c|c|c|}
\hline Link ID & Number of lanes & $t_{0}(\mathrm{~h})$ & Capacity (pch/h) & Link ID & Number of lanes & $t_{0}(\mathrm{~h})$ & Capacity (pch/h) \\
\hline 1 & 3 & 0.022 & 3503.04 & 44 & 3 & 0.009 & 3503.04 \\
\hline 2 & 3 & 0.022 & 3503.04 & 45 & 3 & 0.009 & 3503.04 \\
\hline 3 & 3 & 0.005 & 3503.04 & 46 & 3 & 0.009 & 3503.04 \\
\hline 4 & 3 & 0.005 & 3503.04 & 47 & 3 & 0.018 & 3503.04 \\
\hline 5 & 3 & 0.005 & 3503.04 & 48 & 3 & 0.018 & 3503.04 \\
\hline 6 & 3 & 0.005 & 3503.04 & 49 & 3 & 0.005 & 3503.04 \\
\hline 7 & 3 & 0.024 & 3503.04 & 50 & 3 & 0.005 & 3503.04 \\
\hline 8 & 3 & 0.024 & 3503.04 & 51 & 3 & 0.005 & 3503.04 \\
\hline 9 & 5 & 0.016 & 4723.2 & 52 & 3 & 0.005 & 3503.04 \\
\hline 10 & 5 & 0.016 & 4723.2 & 53 & 3 & 0.005 & 3503.04 \\
\hline 11 & 5 & 0.018 & 5038.08 & 54 & 3 & 0.005 & 3503.04 \\
\hline 12 & 5 & 0.018 & 5038.08 & 55 & 2 & 0.009 & 2634.66 \\
\hline 13 & 5 & 0.009 & 5038.08 & 56 & 2 & 0.009 & 2634.66 \\
\hline 14 & 5 & 0.009 & 5038.08 & 57 & 2 & 0.009 & 2634.66 \\
\hline 15 & 5 & 0.008 & 5038.08 & 58 & 2 & 0.009 & 2634.66 \\
\hline 16 & 5 & 0.008 & 5038.08 & 59 & 3 & 0.005 & 3503.04 \\
\hline 17 & 2 & 0.018 & 2634.66 & 60 & 3 & 0.005 & 3503.04 \\
\hline 18 & 2 & 0.018 & 2634.66 & 61 & 3 & 0.005 & 3503.04 \\
\hline 19 & 2 & 0.012 & 2634.66 & 62 & 3 & 0.005 & 3503.04 \\
\hline 20 & 2 & 0.012 & 2634.66 & 63 & 3 & 0.011 & 3503.04 \\
\hline 21 & 3 & 0.021 & 3721.98 & 64 & 3 & 0.011 & 3503.04 \\
\hline 22 & 3 & 0.021 & 3721.98 & 65 & 3 & 0.008 & 3503.04 \\
\hline 23 & 3 & 0.011 & 3503.04 & 66 & 3 & 0.008 & 3503.04 \\
\hline 24 & 3 & 0.011 & 3503.04 & 67 & 3 & 0.006 & 3503.04 \\
\hline 25 & 2 & 0.015 & 2634.66 & 68 & 3 & 0.006 & 3503.04 \\
\hline 26 & 2 & 0.015 & 2634.66 & 69 & 3 & 0.013 & 3503.04 \\
\hline 27 & 2 & 0.006 & 2634.66 & 70 & 3 & 0.013 & 3503.04 \\
\hline 28 & 2 & 0.006 & 2634.66 & 71 & 2 & 0.009 & 2634.66 \\
\hline 29 & 2 & 0.008 & 2634.66 & 72 & 2 & 0.009 & 2634.66 \\
\hline 30 & 2 & 0.008 & 2634.66 & 73 & 2 & 0.01 & 2634.66 \\
\hline 31 & 2 & 0.014 & 2634.66 & 74 & 2 & 0.01 & 2634.66 \\
\hline 32 & 2 & 0.014 & 2634.66 & 75 & 2 & 0.009 & 2634.66 \\
\hline 33 & 2 & 0.007 & 2634.66 & 76 & 2 & 0.009 & 2634.66 \\
\hline 34 & 2 & 0.007 & 2634.66 & 77 & 2 & 0.017 & 2479.68 \\
\hline 35 & 3 & 0.006 & 3503.04 & 78 & 2 & 0.017 & 2479.68 \\
\hline 36 & 3 & 0.006 & 3503.04 & 79 & 2 & 0.02 & 2634.66 \\
\hline 37 & 3 & 0.005 & 3503.04 & 80 & 2 & 0.02 & 2634.66 \\
\hline 38 & 3 & 0.005 & 3503.04 & 81 & 3 & 0.017 & 3503.04 \\
\hline 39 & 3 & 0.006 & 3503.04 & 82 & 3 & 0.017 & 3503.04 \\
\hline 40 & 3 & 0.006 & 3503.04 & 83 & 2 & 0.009 & 2634.66 \\
\hline 41 & 3 & 0.006 & 3503.04 & 84 & 2 & 0.009 & 2634.66 \\
\hline 42 & 3 & 0.006 & 3503.04 & 85 & 2 & 0.009 & 2634.66 \\
\hline 43 & 3 & 0.009 & 3503.04 & 86 & 2 & 0.009 & 2634.66 \\
\hline
\end{tabular}

TABLE 2: Evacuation and rescue flow demand (Veh/h).

\begin{tabular}{|c|c|c|c|c|c|c|c|c|c|c|}
\hline & \multicolumn{6}{|c|}{ Origins } & \multicolumn{4}{|c|}{ Destinations } \\
\hline & 12 & 13 & 14 & 17 & 18 & 19 & 1 & 4 & 24 & 27 \\
\hline Evacuation flow & 2500 & 2400 & 2200 & 2100 & 3400 & 3200 & -3900 & -6000 & -3500 & -2400 \\
\hline Rescue flow & 1600 & 500 & 400 & 190 & 210 & 200 & -600 & -700 & -900 & -900 \\
\hline
\end{tabular}



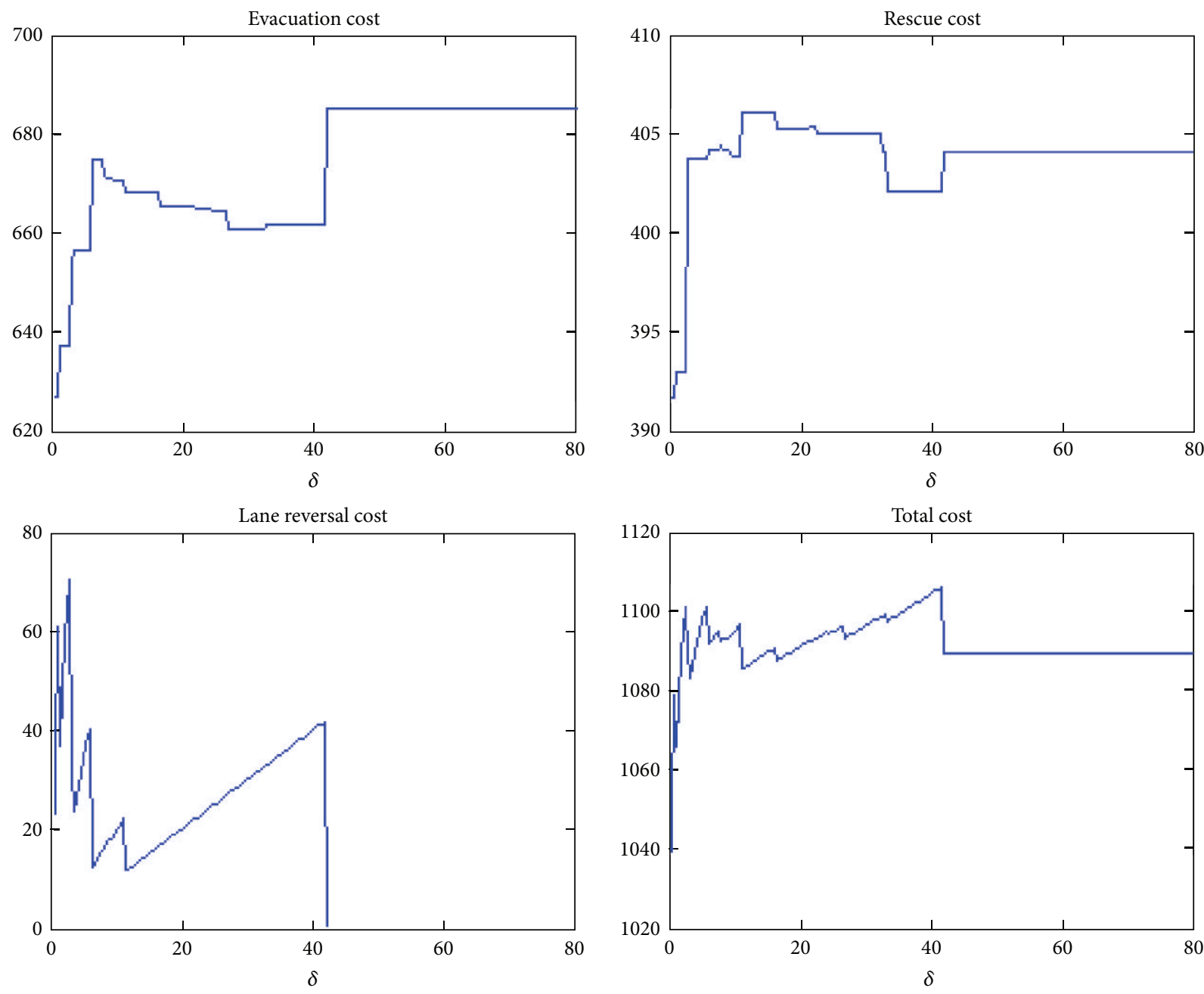

FigURE 3: Results from tests of Group 1.

cannot use contraflow operation to obtain benefits because the reduction of the evacuation cost and rescue cost due to lane reversal operations will not produce an increase of the lane reversal cost. This finding is helpful for emergency managers to control lane reversal costs to a certain extent and obtain benefits from contraflow lane operations.

Group 2. In this group of tests, we do not consider contraflow lane operations, which means that we cannot change the network structure. We aim to investigate how the unit cost of flow conflict affects the evacuation cost, rescue cost, conflict cost, and total cost. The unit evacuation cost $\lambda_{1}$ and unit rescue cost $\lambda_{2}$ take on the same values as in Group 1. The unit flow conflict $\operatorname{cost} \theta$ increases 200 times from 0 in step sizes of 0.000003. The results are shown in Figure 4.

In Figure 4, we observe the following three findings. (1) We find several intervals in which the flow conflict cost increases linearly while the evacuation cost and rescue cost do not change. This result indicates that in these intervals, the linear increase of $\theta$ does not further separate the evacuation flow and rescue flow, and only the flow conflict cost increases linearly. This phenomenon also can be verified by the "total cost" curve in these intervals in which total cost linearly increases due to the linear increase of the conflict cost and the stabilization of the evacuation and rescue costs. (2) Additionally, at the beginning stage of varying unit conflict cost, an interesting phenomenon occurs in that the conflict cost decreases and increases alternately. This result may indicate that when the unit flow conflict cost $\theta$ increases, the flow conflict cost also increases because evacuation flow and rescue flow do not change routes. When $\theta$ increases to a certain extent (we refer to this as a "critical point"), the evacuation flow and rescue flow separate further, and the flow conflict cost decreases. Until $\theta$ is increased to the next critical point, the evacuation cost/rescue cost will remain constant, and the flow conflict cost linearly increases. (3) From the "conflict cost" and "total cost" curves, it can be observed that after certain periods of disturbances of the flow conflict cost and total cost, they increase linearly together. This result may indicate that, at the current evacuation and rescue demand, no matter how large $\theta$ becomes, we cannot obtain a solution that will separate the two types of emergency 

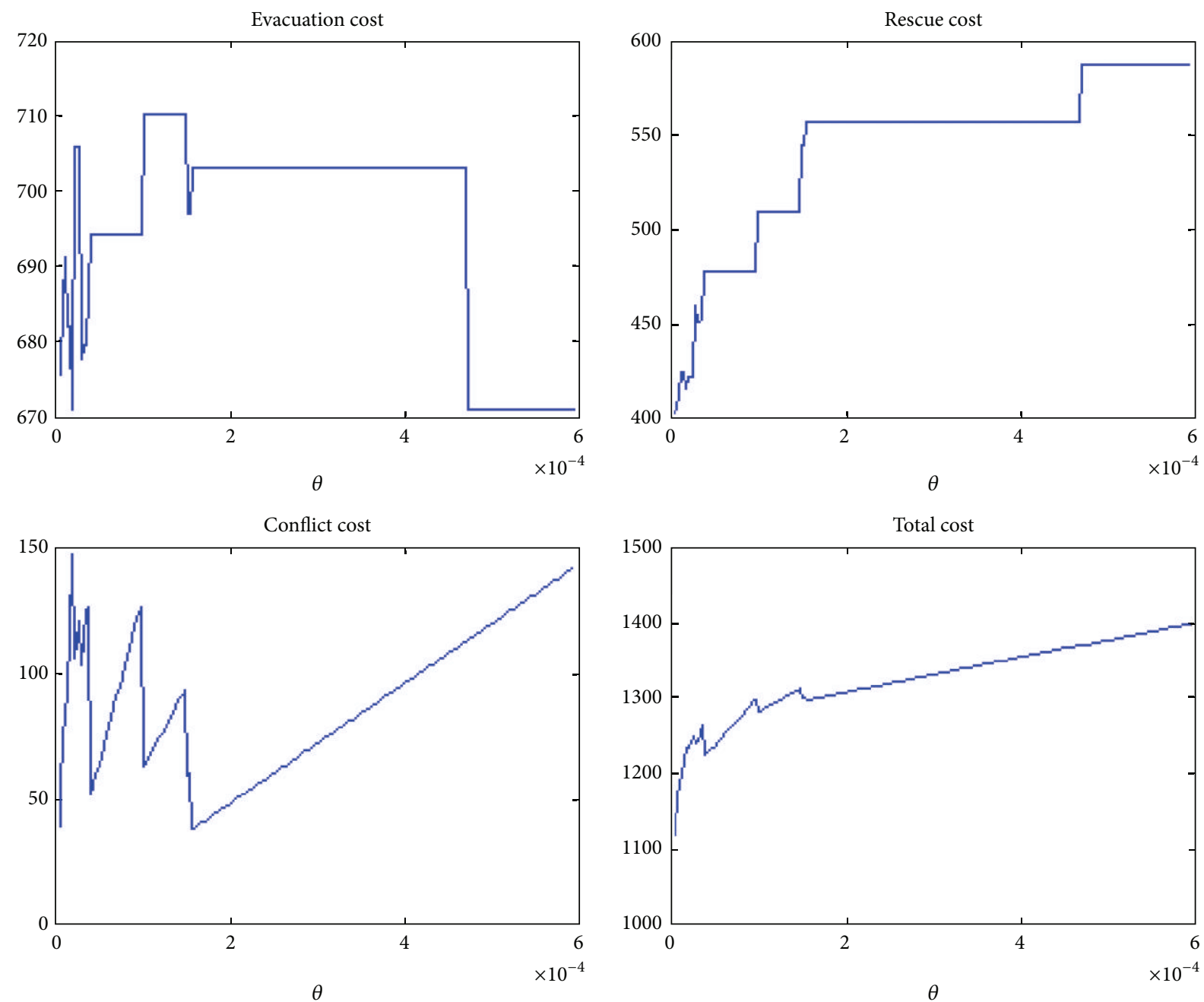

FIGURE 4: Results from tests of Group 2.

flow completely. This observation also inspires us to use lane reversal operations to improve this situation.

Group 3. In this group of tests, we consider contraflow lane operation and flow conflict together. We aim to investigate how the unit cost of flow conflict affects the evacuation cost, rescue cost, conflict cost, contraflow lane operation cost, and total cost. The unit evacuation $\operatorname{cost} \lambda_{1}$ and unit rescue cost $\lambda_{2}$ take on the same values as in Group 1, whereas the unit lane reversal cost $\delta$ is equal to 0.4 . The unit flow conflict cost $\theta$ increases 41 times from 0 in step sizes of 0.000003 . The results are shown in Figure 5.

From the "evacuation and rescue cost" curve, we find that the evacuation cost and rescue cost vary nearly symmetrically. This result is true because, together with the increase in $\theta$, the two types of flow will be separated increasingly further, and one of them will be "pushed out" from the original routes with less travel time to other routes with more travel time to avoid conflict. This observation leads to the fact that if the evacuation cost increases, the rescue cost will decrease and vice versa. From the "conflict cost" curve, it can be observed that the conflict cost stabilizes at zero after $\theta$ increases to a certain extent. This phenomenon is rather interesting because it is completely different than the result from Group 2 in which the conflict cost does not stabilize but increases linearly together with the increase of $\theta$. This observation indicates that the conflict between evacuation flow and rescue flow could be fully avoided using network redesign.

Group 4. In this group of tests, we consider the contraflow lane operation and flow conflict together. We aim to investigate how the unit cost of lane reversal affects the evacuation cost, rescue cost, conflict cost, contraflow lane cost, and total cost. The unit evacuation cost $\lambda_{1}$ and unit rescue cost $\lambda_{2}$ take on the same values as in Group 1, whereas the unit conflict cost $\theta$ is equal to 0.000003 . The unit lane reversal cost $\delta$ increases 41 times from 0.1 in step sizes of 0.4 . The results are shown in Figure 6.

From the "evacuation and rescue cost" curve, we find that the evacuation cost and rescue cost vary nearly with the same trend, which is quite different from the result in Group 3. This observation is reasonable because in Group 3, the variation 

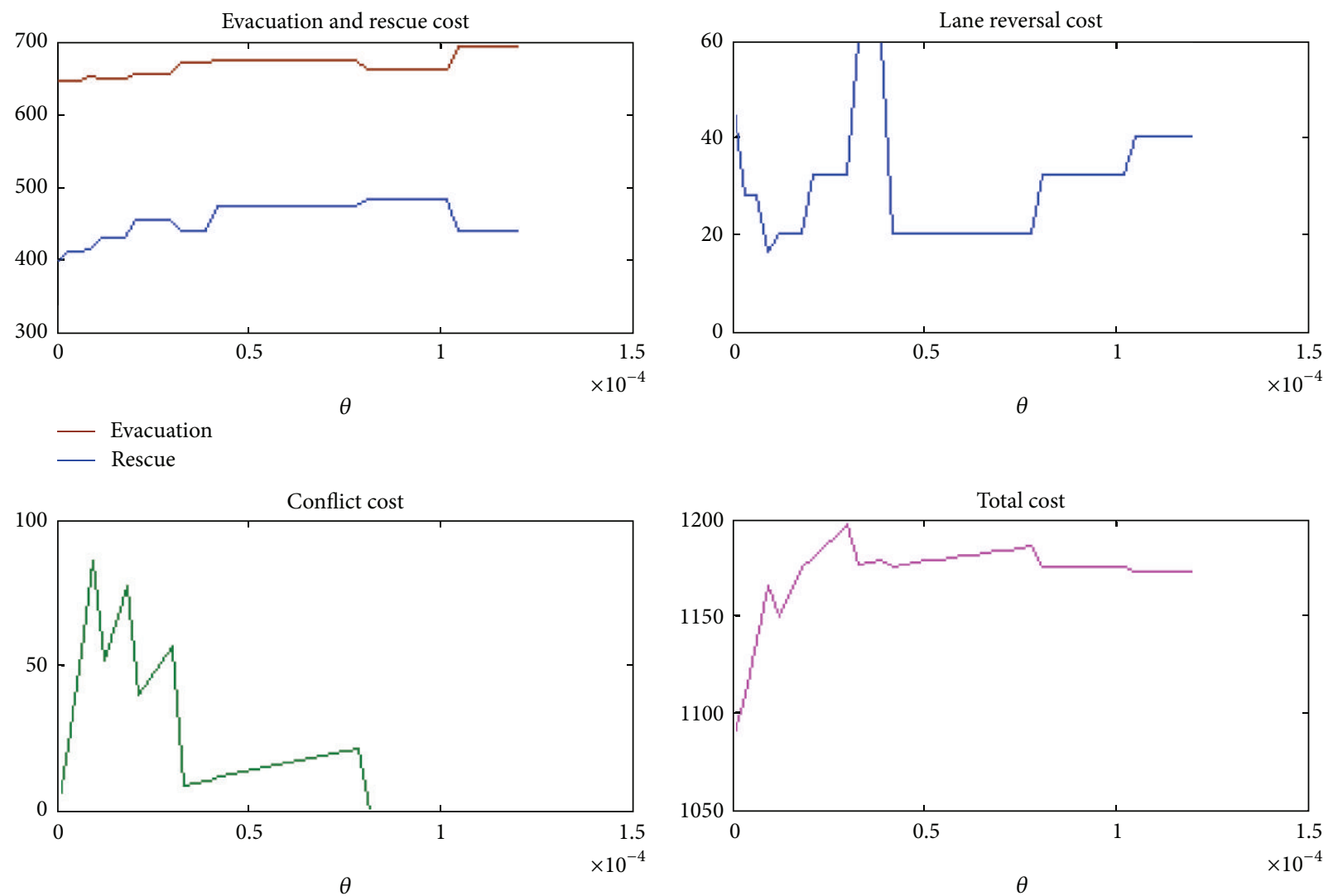

FIgURE 5: Results from tests of Group 3.

of $\theta$ will result in the separation of two flows such that the corresponding costs will change in different directions, but in Group 4, the increase and decrease of number of lane reversals together with the increase of $\delta$ will change the costs related to the two flows in the same direction. It also can be observed that, after $\delta$ becomes slightly larger than 10, the lane reversal cost stabilizes at zero, and the conflict cost, evacuation cost, rescue cost, and total cost stabilize together. The emergency manager can also use this finding to control lane reversal unit cost to maintain the efficiency of contraflow operations.

Selected Managerial Discussions. In emergency management practice, multiple emergency flows should be considered together and collaborate together in the same temporal and spatial framework. Identifying how best to utilize the current road network and route the different types of flow under emergencies in an efficient manner can be a challenging problem. The critical question related to this situation is how to determine the unit values of the four components of cost because the different values that they take on can lead to obviously different decision. For example, if the decision-makers emphasize no conflict between multiple flows, this means that $\theta$ will be relatively large, the capacity of the links will likely not be fully explored because various types of flow will independently occupy certain links to avoid conflict among them, and certain links may still contain much capacity. If $\theta$ takes on a relatively small value, which occurs if managers do not pay much attention to conflict, this will lead to an absolutely different routing plan and contraflow operation strategy. Therefore, in practice, decision-makers should pay careful attention to deciding on the values of various unit costs to obtain a valuable and practical emergency solution.

Another interesting point is that the model presented in this research can be used in emergency flow cooperation scenarios but also can be used in other fields. A typical example is the assumption that a pipe network will be used for transportation of multiple materials. Certain materials are not allowed to conflict with each other or chemical reactivity will occur and cause damage, whereas other materials are allowed to conflict to a certain extent, but this will lead to an additional cost for separating the materials. The problem of how to decide on a plan to use the current pipe network to transport these materials is quite similar to the problem presented in this research.

\section{Conclusions}

This work studies a multiple emergency flow collaboration and optimal contraflow design (MEFR) problem in an urban highway network. A nonconvex mixed-integer nonlinear programming model is developed to describe this problem, which integrates four components of emergency cost: 

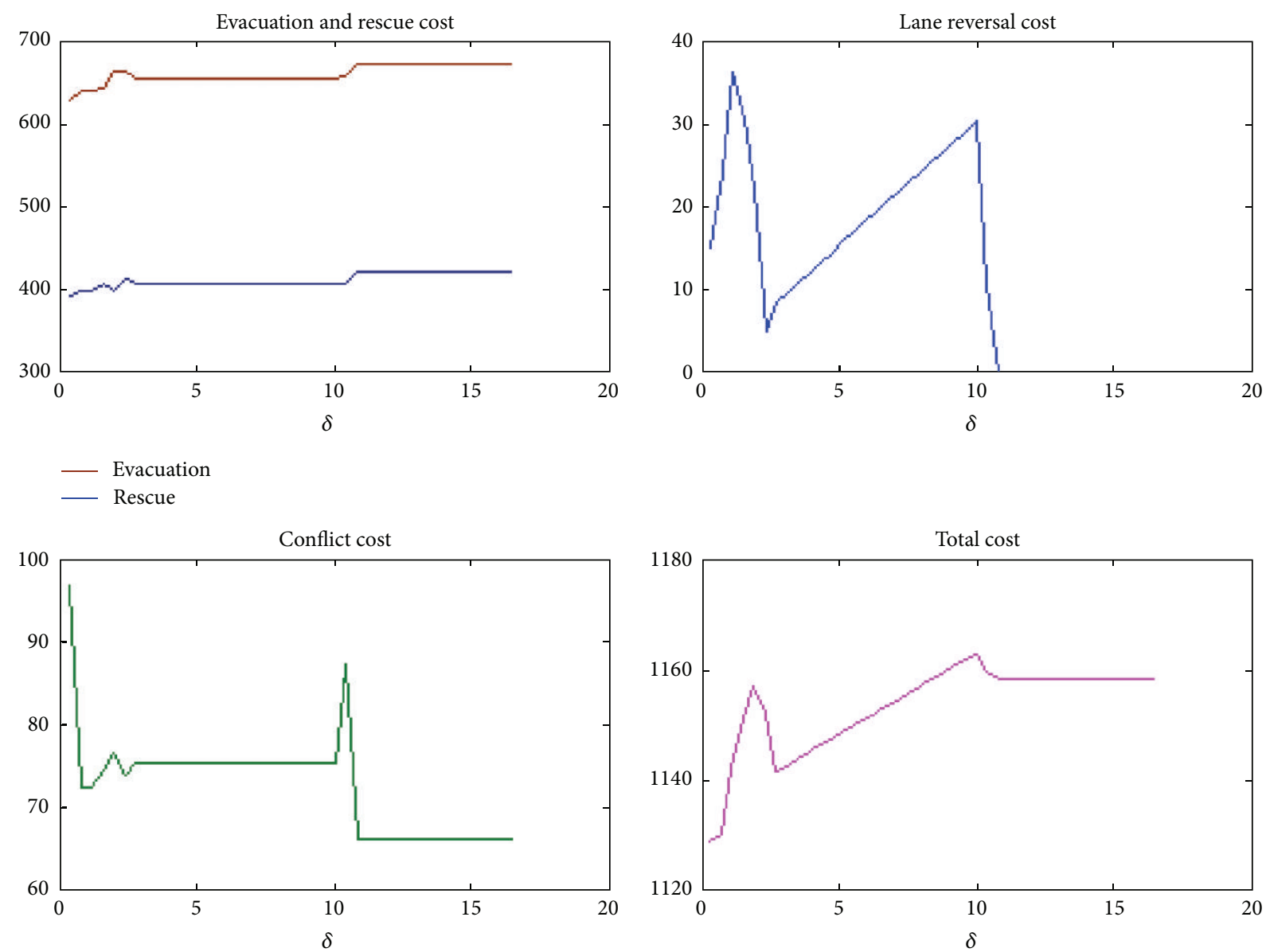

FIGURE 6: Results from tests of Group 4.

evacuation time cost, rescue time cost, flow conflict cost, and contraflow lane operation cost. Because this model is relatively difficult to solve, we use the GAMS/BARON solver to obtain solutions. Case studies in the downtown area of Harbin City in China are conducted to illustrate how our model operates. Various managerial insights are also drawn from the results as follows: (i) decision-makers should pay careful attention to determination of the values of various unit costs because these decisions will lead to completely different emergency strategies; (ii) decision-makers should properly control the lane reversal cost to guarantee that the operation of contraflow will produce a positive benefit; and (iii) different values of unit conflict cost can be used to adjust the separation level of multiple emergency flows.

In future work, an effective algorithm should be developed to solve the nonconvex MINLP model presented in this research. The solution of this model is relatively time consuming with an average cost of 15 minutes required to solve the model with BARON. Certain heuristic algorithms should be developed to achieve good balance between solution precision and solution efficiency.

\section{Notation Summary}

$G=(A, V)$ : A directed network with link set $A$ and node set $V$

$\mathrm{O}_{e}^{*}: \quad \quad \quad$ Virtual evacuation origin node

$D_{e}^{*}: \quad$ Virtual evacuation destination node

$\mathrm{O}_{e}$ : $\quad$ Real-world evacuation origin node set;

$D_{e}: \quad$ Real-world evacuation destination node set

$\mathrm{O}_{r}^{*}: \quad \quad$ Virtual rescue origin node

$D_{r}^{*}: \quad$ Virtual rescue destination node

$\mathrm{O}_{r}$ : $\quad$ Real-world rescue origin node set

$D_{r}: \quad$ Real-world rescue destination node set

$x_{i j}$ : $\quad$ Evacuation flow on link $(i, j) \in A$ (decision variable)

$y_{i j}: \quad$ Rescue flow on link $(i, j) \in A$ (decision variable)

$\gamma_{i j}: \quad \quad \quad$ Number of lanes of link $(i, j) \in A$ after optimal contraflow design (decision variable)

$\mathrm{NL}_{i j}: \quad \quad$ Number of lanes of link $(i, j) \in A$ before optimal contraflow design 


\begin{tabular}{|c|c|}
\hline$t_{i j}^{0}:$ & The free flow travel time of link $(i, j) \in A$ \\
\hline$t_{i j}:$ & Travel time of link $(i, j) \in A$ \\
\hline$\mu_{i j}$ & $\begin{array}{l}\text { Capacity of link }(i, j) \in A \text { before optimal } \\
\text { contraflow design }\end{array}$ \\
\hline $\bar{\mu}_{i j}:$ & $\begin{array}{l}\text { Capacity of link }(i, j) \in A \text { after optimal } \\
\text { contraflow design }\end{array}$ \\
\hline$\xi(i), \zeta(i)$ & $\begin{array}{l}\text { The downstream nodes set and upstream } \\
\text { nodes set of node } i \in V\end{array}$ \\
\hline$S_{j}^{e, o}:$ & $\begin{array}{l}\text { Sending flow demand of evacuation origin } \\
\text { node } j \in O_{e}\end{array}$ \\
\hline$R_{j}^{e, d}:$ & $\begin{array}{l}\text { Receiving flow demand of evacuation } \\
\text { destination node } j \in D_{e}\end{array}$ \\
\hline$S_{j}^{r, O}:$ & $\begin{array}{l}\text { Sending flow demand of rescue origin } \\
\text { node } j \in O_{r}\end{array}$ \\
\hline$R_{j}^{r, d}:$ & $\begin{array}{l}\text { Receiving flow demand of rescue } \\
\text { destination node } j \in D_{r}\end{array}$ \\
\hline$\lambda_{1}:$ & $\begin{array}{l}\text { Generalized cost of unit evacuation flow } \\
\text { travel time }\end{array}$ \\
\hline$\lambda_{2}:$ & $\begin{array}{l}\text { Generalized cost of unit rescue flow travel } \\
\text { time }\end{array}$ \\
\hline$\theta:$ & $\begin{array}{l}\text { Generalized cost of the conflict between } \\
\text { unit rescue flow and unit evacuation flow }\end{array}$ \\
\hline$\delta:$ & Generalized cost of the one lane reversal \\
\hline$d_{1}:$ & $\begin{array}{l}\text { Total evacuation demand flow; that is, } \\
d_{1}=\sum_{j \in O_{e}} S_{j}^{e, o}=\sum_{j \in D_{e}} R_{j}^{e, o}\end{array}$ \\
\hline$d_{2}:$ & $\begin{array}{l}\text { Total rescue demand flow; that is, } \\
d_{2}=\sum_{j \in O_{r}} S_{j}^{r, o}=\sum_{j \in D_{r}} R_{j}^{r, o}\end{array}$ \\
\hline & Coefficients of the BPR function. \\
\hline
\end{tabular}

\section{Conflict of Interests}

The authors declare that there is no conflict of interests regarding the publication of this paper.

\section{Acknowledgments}

This research is supported by the National Natural Science Foundation of China (Project no. 71203045), Heilongjiang Natural Science Foundation (Project no. E201318), and the Fundamental Research Funds for the Central Universities (Grant no. HIT.KISTP.201421). This work was performed at the Key Laboratory of Advanced Materials \& Intelligent Control Technology on Transportation Safety, Ministry of Communications, China.

\section{References}

[1] S. Bretschneider and A. Kimms, "A basic mathematical model for evacuation problems in urban areas," Transportation Research A: Policy and Practice, vol. 45, no. 6, pp. 523-539, 2011.

[2] J. Ford and D. R. Fulkerson, "Maximal flow through a network," Canadian Journal of Mathematics, vol. 8, no. 3, pp. 399-404, 1956.

[3] D. Gale, "Transient flows in networks," Michigan Mathematical Journal, vol. 6, no. 1, pp. 59-63, 1959.

[4] L. G. Chalmet, R. L. Francis, and P. B. Saunders, "Network models for building evacuation," Fire Technology, vol. 18, no. 1, pp. 90-113, 1982.
[5] H. W. Hamacher and S. Tufekci, "On the use of lexicographic min cost flows in evacuation modeling," Naval Research Logistics, vol. 34, pp. 487-503, 1987.

[6] W. Choi, H. W. Hamacher, and S. Tufekci, "Modeling of building evacuation problems by network flows with side constraints," European Journal of Operational Research, vol. 35, no. 1, pp. 98$110,1988$.

[7] C. E. Dunn and D. Newton, "Optimal routes in GIS and emergency planning applications," Area, vol. 24, no. 3, pp. 259267, 1992.

[8] T. J. Cova and J. P. Johnson, "A network flow model for lanebased evacuation routing," Transportation Research A: Policy and Practice, vol. 37, no. 7, pp. 579-604, 2003.

[9] E. Miller-Hooks and S. S. Patterson, "On solving quickest time problems in time-dependent, dynamic networks," Journal of Mathematical Modelling and Algorithms, vol. 3, no. 1, pp. 39-71, 2004.

[10] S. Opasanon, On finding paths and flows in multicriteria, stochastic and time-varying networks [Ph.D. dissertation], University of Maryland, 2004.

[11] G. Ayfadopoulou, I. Stamos, E. Mitsakis, and J. Grau, "Dynamic traffic assignment based evacuation planning for CBD areas," Procedia-Social and Behavioral Sciences, vol. 48, pp. 1078-1087, 2012.

[12] C. Xie, D.-Y. Lin, and S. Travis Waller, "A dynamic evacuation network optimization problem with lane reversal and crossing elimination strategies," Transportation Research E: Logistics and Transportation Review, vol. 46, no. 3, pp. 295-316, 2010.

[13] H. S. Mahmassani, H. Sbayti, and X. Zhou, DYNASMART-P Version 1.0 User's Guide, Maryland Transportation Initiative, University of Maryland, 2004.

[14] M. Ben-Akiva, D. Mcfadden, K. Train, J. Walker, C. Bhat, and M. Bierlaire, Development of a Deployable Real-Time Dynamic Traffic Assignment System: DynaMIT And DynaMIT$P$ User's Guide, Intelligent Transportation Systems Program, Massachusetts Institute of Technology, 2002.

[15] L. C. MacDorman, "Case study in sensitivity of highway economic factors," Highway Research Record, vol. 100, pp. 2-19, 1965.

[16] T. S. Glickman, Optimal periodic control of reversible traffic operations [Ph.D. dissertation], Johns Hopkins University, 1970.

[17] J. Hemphill and V. H. Surti, "A feasibility study of a reversiblelane facility for a Denver street corridor," Transportation Research Record, vol. 514, pp. 29-32, 1974.

[18] R. J. Caudill and N. M. Kuo, "Development of an interactive planning model for contraflow lane evaluation," Transportation Research Record, pp. 47-54, 1983.

[19] E. Urbina and B. Wolshon, "National review of hurricane evacuation plans and policies: a comparison and contrast of state practices," Transportation Research A: Policy and Practice, vol. 37, no. 3, pp. 257-275, 2003.

[20] P. B. Wolshon and L. Lambert, Convertible Roadways and Lanes: A Synthesis of Highway Practice, vol. 340 of NCHRP Synthesis, National Cooperative Highway Research Program, Transportation Research Board, Washington, DC, USA, 2004.

[21] H. Tuydes and A. Ziliaskopoulos, "Tabu-based heuristic approach for optimization of network evacuation contraflow," Transportation Research Record, no. 1964, pp. 157-168, 2006.

[22] S. Kim, S. Shekhar, and M. Min, "Contraflow transportation network reconfiguration for evacuation route planning," IEEE Transactions on Knowledge and Data Engineering, vol. 20, no. 8, pp. 1115-1129, 2008. 
[23] G. L. Hamza-Lup, K. A. Hua, and R. Peng, "Leveraging etransportation in real-time traffic evacuation management," Electronic Commerce Research and Applications, vol. 6, no. 4, pp. 413-424, 2007.

[24] Q. Meng, H. L. Khoo, and R. L. Cheu, "Microscopic traffic simulation model-based optimization approach for the contraflow lane configuration problem," Journal of Transportation Engineering, vol. 134, no. 1, pp. 41-49, 2008.

[25] C. Xie and M. A. Turnquist, "Lane-based evacuation network optimization: an integrated Lagrangian relaxation and tabu search approach," Transportation Research C: Emerging Technologies, vol. 19, no. 1, pp. 40-63, 2011.

[26] C. Xie and M. A. Turnquist, "Integrated evacuation network optimization and emergency vehicle assignment," Transportation Research Record, no. 2091, pp. 79-90, 2009.

[27] F. Yuan, A proposed framework for simultaneous optimization of evacuation traffic distribution and assignment [Ph.D. dissertation], University of Tennessee, 2005.

[28] L. R. Ford and D. R. Fulkerson, Flows in Network, Princeton University Press, Princeton, NJ, USA, 1962.

[29] F. S. Hillier and G. J. Lieberman, Introduction to Operations Research, McGraw-Hill, New York, NY, USA, 1990.

[30] S. C. Dafermos, "Sparrow the traffic assignment problem for a general network," Journal of Research of the National Bureau of Standards B, vol. 73, pp. 91-118, 1969.

[31] Y. Liu, An integrated optimal control system for emergency evacuation [Ph.D. dissertation], University of Maryland, 2007.

[32] R. Östermark, "Concurrent processing of mixed-integer nonlinear programming problems," Kybernetes, vol. 38, no. 6, pp. 966-989, 2009. 


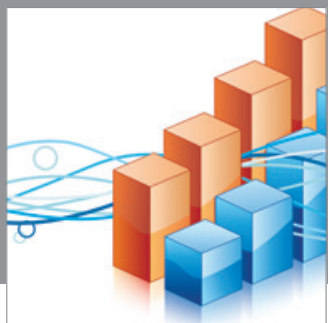

Advances in

Operations Research

mansans

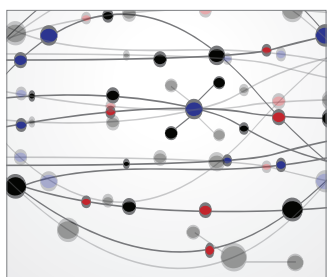

The Scientific World Journal
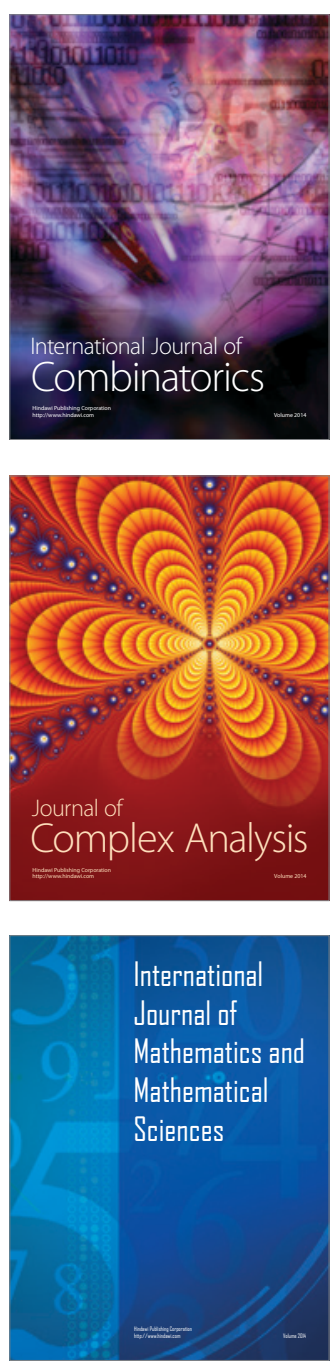
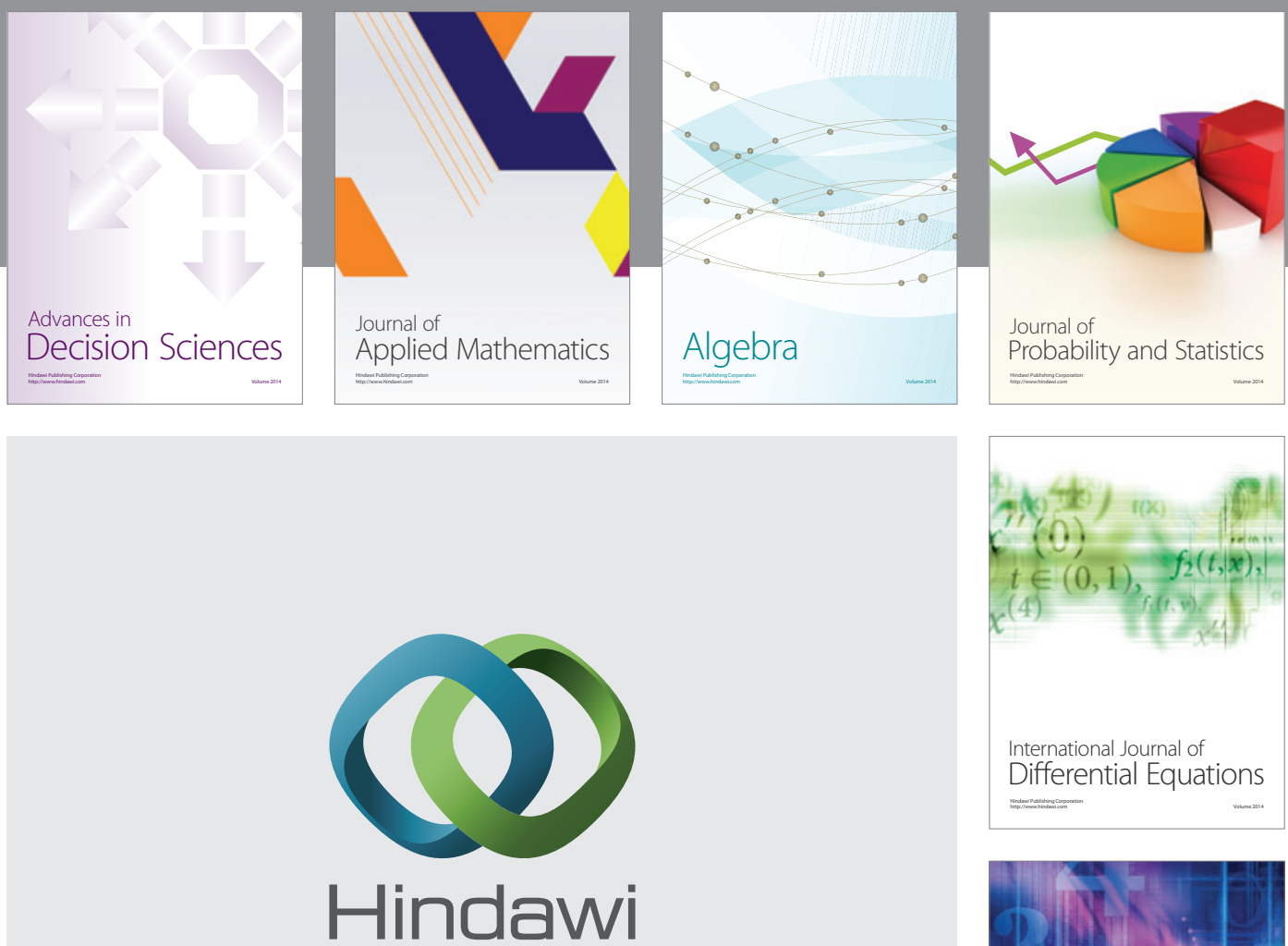

Submit your manuscripts at http://www.hindawi.com
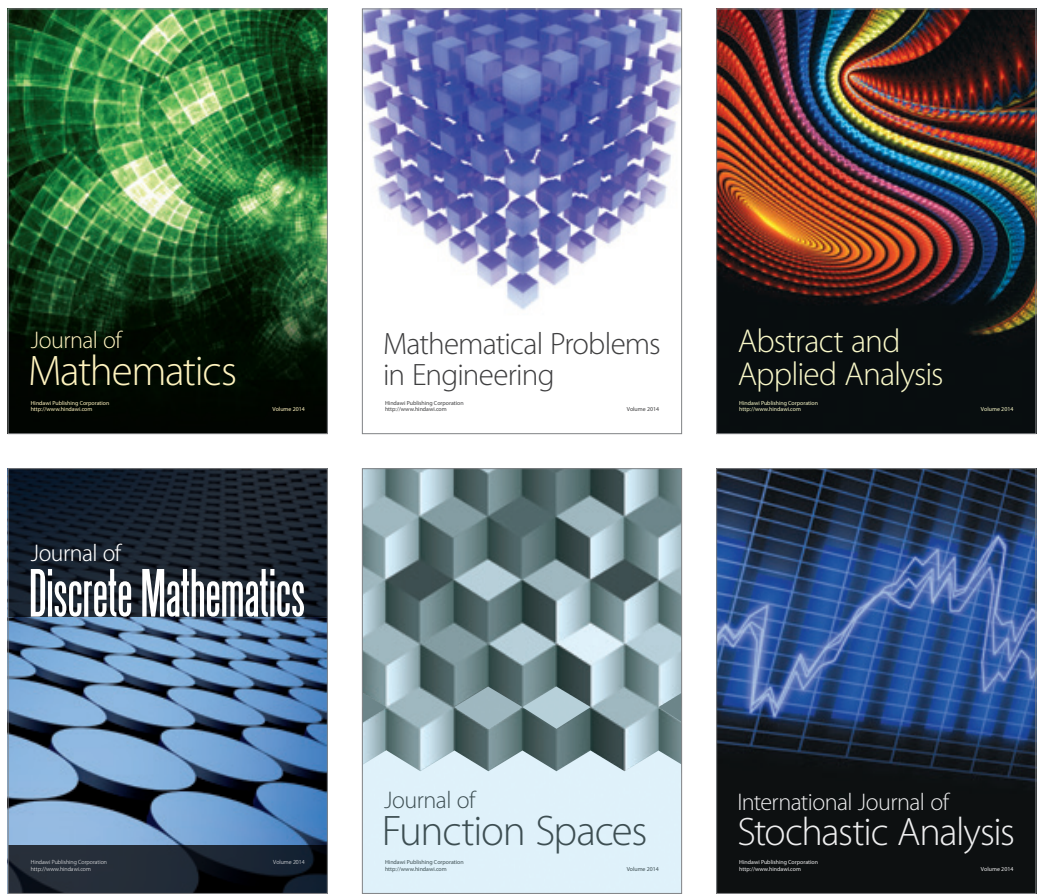

Journal of

Function Spaces

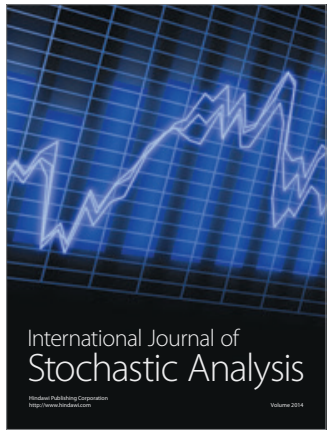

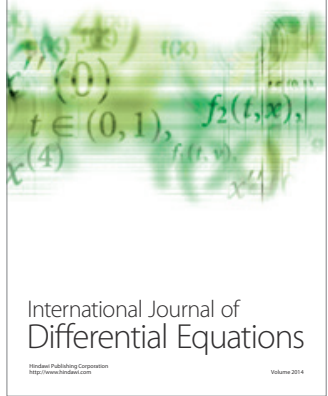
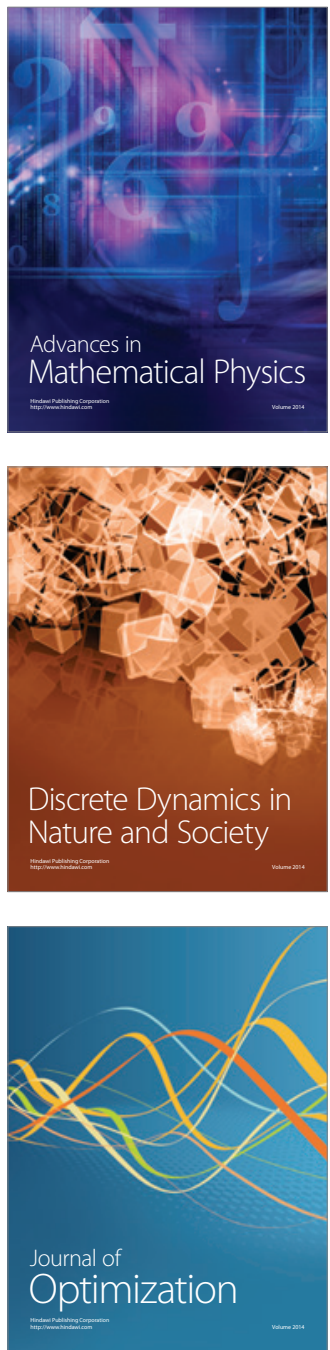